\title{
Optimal Design of Photovoltaic-Battery Systems Using Interval Type-2 Fuzzy Adaptive Genetic Algorithm
}

\author{
Ontoseno Penangsang, Muhammad Abdillah, Rony Seto Wibowo, Adi Soeprijanto \\ Department of Electrical Engineering, Institut Teknologi Sepuluh Nopember (ITS), Surabaya, Indonesia \\ Email: zenno_376@yahoo.com, abdillah@elect-eng.its.ac.id,ronyseto@yahoo.com, adisup@ee.its.ac.id
}

Received 2013

\begin{abstract}
Many countries have been triggered to provide a new energy policy which promotes renewable energy applications because of public awareness to reduce the global warming and rising in fuel prices. Renewable energy sources such as solar energy are green and promising energy in the future for widespread use. Combining renewable energy sources with battery makes electricity supply more economical and reliable to meet all possible load level. This paper proposed a new hybrid method to optimize Photovoltaic (PV)-Battery systems. The proposed method was named Interval type-2 fuzzy adaptive genetic algorithm (IT2FAGA). Genetic algorithm (GA) is one of modern optimization techniques that has been successfully applied in various areas of power systems. To enhance the ability of GA to prevent trapping in local optima and increase convergence in a global optima, the crossover probability $\left(p_{\text {cross }}\right)$ and the mutation probability $\left(p_{m u t}\right)$, parameters in GA, are tuned using interval type-2 fuzzy logic (IT2FL). Objective function used in this paper was the annual cost of sytem (ACS) consisting of the annual capital cost (ACC), annual replacement cost (ARC), annual operation cost maintenance (AOM). The proposed method was also compared to fuzzy adaptive genetic algorithm (FGA) and standard genetic algorithm (SGA). Simulation results indicated that the proposed method had a better perfomance in minimizing the objective function than the other two methods.
\end{abstract}

Keywords: Photovoltaic (PV); Battery; GA; IT2FL; IT2FAGA

\section{Introduction}

The use of renewable energy resources has mapped in many developed countries. One of the most promising technologies among renewable energy resources is Photovoltaic (PV). PV technology is growing rapidly in developed countries and developing countries. An optimal number of solar cell panels and battery storages are very essential in the design of PV-Battery systems applied in remote rural areas. The appropriate control strategy is required in designing PV-battery systems. Design of PV-battery system is a complicated task and it needs a mathematical model involving a significant number of variables. To solve such kind of problem, artificial intelligence (AI) technique is very powerful. Nowadays, AI techniques are becoming a popular technique to solve complicated practical problems in various fields [1]. One of the most commonly used of the AI techniques is genetic algorithm [2]. In recent decades, genetic algorithms (GA) are very popular algorithms to solve optimization problems because of robustness in finding the optimal solution [3]. The genetic algorithm is faster in finding the best solution than other random searching methods. However, the performance of standards GA significantly depends on the algorithm pa- rameters. In addition, solution of GA sometimes gets stuck in local optima. Therefore, to avoid this problem, this study proposed hybrid algorithm called interval type-2 fuzzy adaptive genetic algorithm (IT2AGA). Interval type-2 fuzzy logic (IT2FL) was used to adjust the crossover probability $\left(p_{\text {cross }}\right)$ and the mutation probability $\left(p_{m u t}\right)$ in order to improve the performance of genetic algorithm. IT2FL has been widely applied in many fields, and the results are very good [4-7].

The proposed method in this study was used to optimize PV-battery systems in minimizing the annual cost of system (ACS). The paper is organized as follows: Section II presents a brief review of the materials and methods. The proposed method is described in Section III. Part IV presents the implementation of proposed method. Simulation and result are described in Chapter V. The last section is the conclusion.

\section{Materials and Methods}

\subsection{Photovoltaic (PV)}

Equation (1) describes a simple model of PV panels. Conditions of insolation and area of PV panel greatly affect the power generated by PV panels [8]. 


$$
P_{P V}(t)=\eta \times A_{p} \times N_{P V} \times E(t)
$$

where $\eta$ is energy conversion efficiency $(\%) ; A_{p}$ is area of PV panel; $N_{P V}$ is the number of PV panel; $E(t)$ is insolation data $\left(\mathrm{w} / \mathrm{m}^{2}\right)$

\subsection{Battery}

Equation (2) shows the power generated at the time $t$ of the PV panel.

$$
P_{G}(t)=P_{P V}(t)
$$

when the total power output of PV panels is greater than the load demand, battery charges, and the number of battery charging at the time $t$ can be expressed in Equation (3).

$$
P_{B A T}(t)=P_{B A T}(t-1)+\left(P_{G}(t)-P_{L}(t)\right)
$$

when the total power output of PV panels is less than the load demand, the battery discharges, and the number of battery discharging at the time $t$ can be expressed in Equation (4).

$$
P_{B A T}(t)=P_{B A T}(t-1)-\left(P_{L}(t)-P_{G}(t)\right)
$$

where $P_{B A T}(t)$ is battery capacity at time $t, P_{B A T}(t-1)$ is battery capacity at an earlier time or $t-1$.

\subsection{System Configuration}

The system configuration model in this study was composed of PV panel and battery connected to the load demand through the inverter. The system configuration model used in this study is described in Figure 1.

\subsection{Operational Strategy}

The concept of operational strategy for PV-battery system is described as follows:

1. When load demand $\left(P_{L}(t)\right)$ is smaller than output power generated by $\mathrm{PV}$ panels $\left(P_{P V}(t)\right)$, excess power is used to charge the battery.

2 . When excess power is higher than the inverter capacity, battery are filled equally to inverter capacity and the surplus power is discarded.

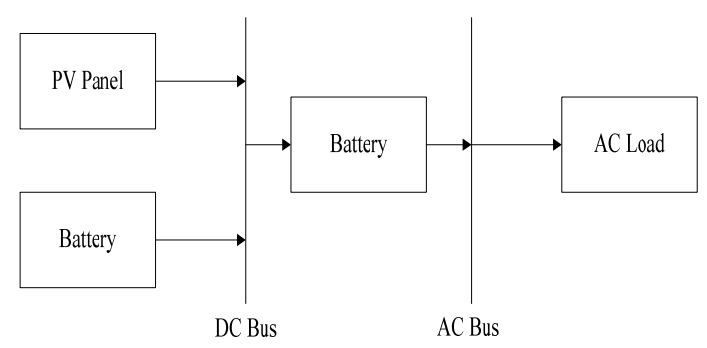

Figure 1. System configuration.
3. When load demand $\left(P_{L}(t)\right.$ is greater than $\left.P_{P V}(t)\right)$ and $P_{B A T}(t)$ of battery are higher than $\mathrm{P}_{\mathrm{BATmin}}$ then lack of power will be supplied by battery.

4. When lack of power is higher than inverter capacity, battery is discharged equally to inverter capacity

\subsection{Problem Formulation}

\subsubsection{Objective Function}

Objective function used in this study for optimizing of PV-battery system was the Annual Cost of System (ACS) consist of the Annual Capital Cost (ACC), Annual Replacement Cost (ARC), and Annual Operation Cost Maintenance (AOM) expressed in equation (5) [8].

$$
A C S=A C C+A O M+A R C
$$

Annual Capital Cost (ACC) of each unit is represented using Equation (6) [8].

$$
A C C=C_{c a p} \times C R F(i, Y)
$$

where $C_{c a p}$ is capital cost of each component (US\$), $Y$ is duration project (year). CRF is capital recovery factor, ratio to calculate the present value of a series of equal annual cash flow.

Annual Maintenance Cost (AOM) of each unit as a function of interest rates and duration project is expressed in Equation (7) [8].

$$
A O M=\frac{A O M(1) \times(1+f)^{Y} \text { project }}{Y_{\text {project }}}
$$

where $f$ is annual inflation rate (\%)

Annual Replacement Cost (ARC) is the replacement cost of each unit during time period of the project and it is calculated using Equation (8) [8].

$$
A R C=C_{\text {rep }} \times \operatorname{SFF}\left(i, Y_{\text {rep }}\right)
$$

where $C_{\text {rep }}$ is replacement cost per unit (battery) (US\$). $Y_{\text {rep }}$ is duration of each unit (year). SFF is sinking fund factor, rasio to calculate the future value of a series of equal annual cash flow.

\subsubsection{Constrain}

Power balance constraint for each period time $t$, total power of PV-battery systems, must supply the load $\left(P_{L}\right)$ demand with a certain reliability criterion. This relationship can be represented by,

$$
P_{P V}+P_{B A T} \geq P_{L}
$$

Constraints of the PV panels and batteries

$$
N_{P V}, N_{B A T} \geq 0
$$

Constraints of battery capacity

$$
P_{B A T \text { min }} \leq P_{B A T} \leq P_{B A T \max }
$$




\section{Proposed Method}

The discussion in this section is described briefly on genetic algorithm, interval type-2 fuzzy logic and interval type-2 fuzzy adaptive genetic algorithm:

\subsection{Genetic Algorithm}

Genetic algorithm is a part of the evolutionary algorithms family, computational models inspired by nature. Genetic algorithm is a powerful stochastic search algorithm based on the mechanism of natural selection and natural genetics. Flowchart of the GA is shown in Figure 2.

Genetic algorithm is applied to optimize the parameters of a very complex system which is difficult to be solved by conventional methods. Genetic algorithm maintains a set of candidate solutions called population and it repeatedly modifies them. In each generation, Genetic Algorithm selects individuals from the current population to be parents and uses them to produce children for the next generation. Candidate solutions are represented as strings called chromosomes. A fitness function is used to reflect how well the value of each member of the population is.

Genetic algorithm operates in cycles called generations, expressed as follows,

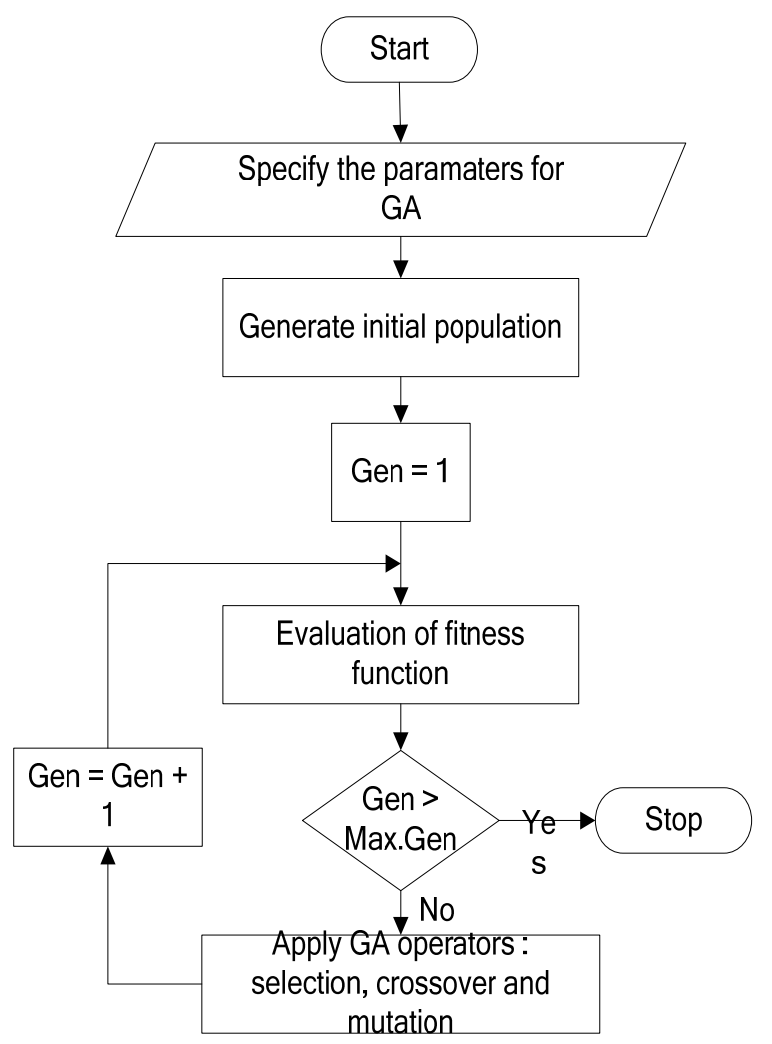

Figure 2. Flowchart of GA. a. An initial population of individuals is randomly generated.

b. Every member of population is evaluated using the fitness function.

c. Based on the fitness value, some individuals will be selected for the next generation. Selected individuals will be combined through the process of crossover by exchanging genetic information between pairs of individuals contained in the current population. After that, each individual in the population will be mutated with a given probability, through random processes replacing one gene with another to produce a new genetic structure.

\subsection{Interval Type $\mathbf{2}$ Fuzzy Logic (IT2FL)}

The concept of interval type-2 fuzzy set was introduced by Zadeh [9] as an extension of the usual concept of fuzzy set, ie, type-1 fuzzy set. Explanation of interval type 2 fuzzy logic (IT2FL) is as follows,

Similar to type-1 fuzzy logic, interval type-2 fuzzy logic has fuzzifier, knowledge base, inference engine, and the output processor. The output processor consists of type reducer and defuzzifier. It generates a type-1 fuzzy set output (from the type-reducer) or a crisp number (from the defuzzifier). The basic structure of type-2 fuzzy logic is shown in Figure $\mathbf{3}$ is as follows.

a. Fuzzifier: It converts the input (real value) into the values of fuzzy membership functions.

b. Inference System: Fuzzy reasoning mechanisms are applied by the interval type-2 FL to get the output fuzzy.

c. Defuzzifiier/ type reducer: Defuzzifier function is to convert the fuzzy output into a precise value, while the function of the type reducer is to transform the interval type-2 FL set to type-1 fuzzy set.

d. Knowledge Base: In this section, it consists of a fuzzy set rule called set of basic rules and membership functions called the database.

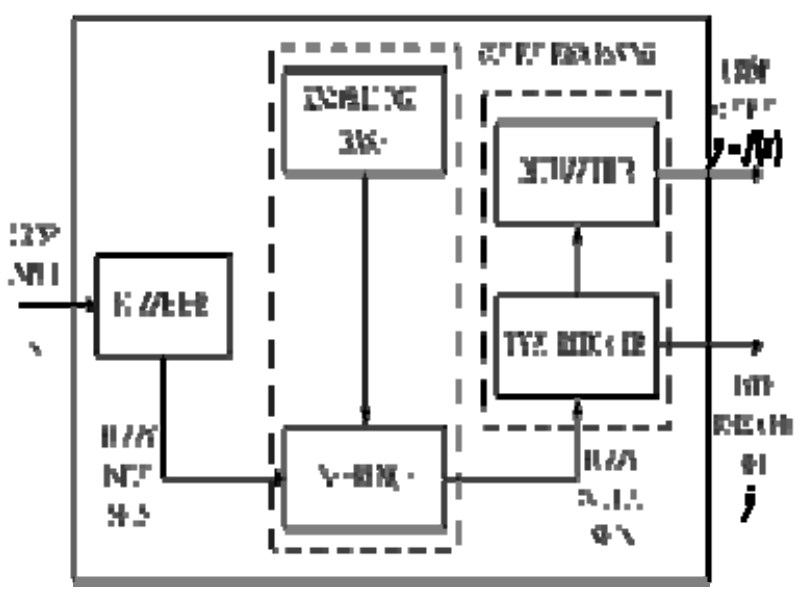

Figure 3. The structure of interval type-2 fuzzy logic. 


\subsection{Adaptive Interval Type 2 Fuzzy Logic Genetic Algorithm}

In this study, two parameters of GA are the probability of crossover $\left(p_{\text {cross }}\right)$ and mutation probability $\left(p_{\text {mut }}\right)$ to be varied dynamically during the generation. IT2FL was used to improve the performance of GA in order to be optimal and efficient. Membership functions and the rule base were the basic criteria that determine the performance IT2FL. Defining these parameters according to the characteristics of the system has significant meanings to achieve optimal performance should be considered in the IT2FL design procedure. Membership functions for the Best Fitness, crossover probability $\left(p_{\text {cross }}\right)$ and mutation probability $\left(p_{m u t}\right)$ consisted of fuzzy set LOW, MEDIUM and HIGH. To determine the IT2FL rules, BestFitness was used as input of IT2FL, while the IT2FL outputs were the crossover probability $\left(p_{\text {cross }}\right)$ and the mutation probability $\left(p_{m u t}\right)$. For IT2FL rule, Mamdani type was used to formulate the conditional statement of IT2FL rules.

Figure 4 shows that the input and output variables of Best Fitness, $p_{\text {cross }}$ and $p_{m u t}$ are used as IT2FL fuzzification. Two parameters of GA, $p_{\text {cross }}$ and $p_{m u t}$ vary based on the fitness function value according to the following logic [10],

a. Best fitness value for each generation is expected to change over several generations. Yet, if it doesn't change significantly over a few generations, it is necessary to make changes on the probability of crossover $\left(p_{\text {cross }}\right)$ and mutation probability $\left(p_{m u t}\right)$.

b. The diversity of population is one of the factors that affect in searching the optimum value. The variation of the fitness value of the objective function of a population is a measure of its diversity. Therefore, $p_{m u t}$ and $p_{\text {cross }}$ can be changed to get good results.

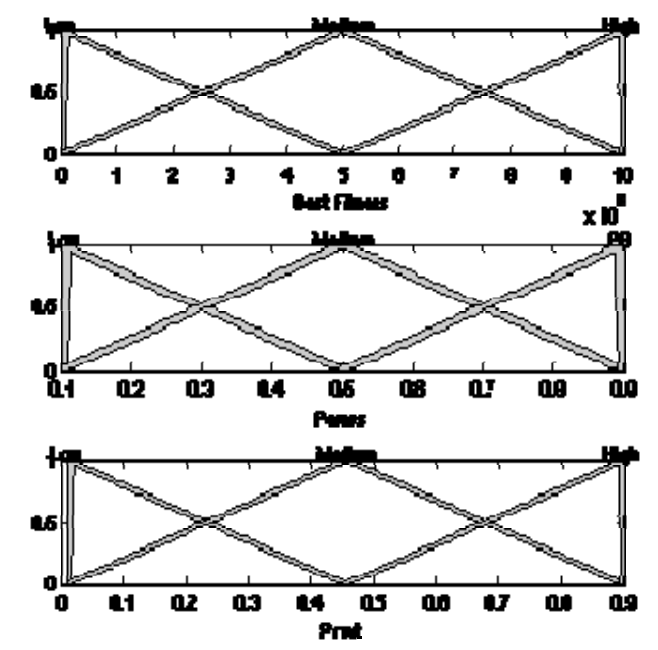

Figure 4. Input and output membership function of IT2FL. c. For example, if Best Fitness is LOW, then ( $p_{\text {cross }}$ is HIGH) and ( $p_{m u t}$ is LOW). The rules of IT2FL is used to tune $p_{\text {cross }}$ and $p_{\text {mut }}$ as shown in Table $\mathbf{1}$. The defuzzification method which used in IT2FL design is centroid method.

\section{Implementation of Proposed Method}

In this section, the application of the proposed method for optimizing the PV-battery systems are expressed as follows

1. Input data which included annual meteorological data, parameters of PV panels, inverters, batteries and load demand.

2. Initialize the population: Initial a number of chromosomes consisted of two genes $x_{i d}=\left[x_{i l}, x_{i 2}, \ldots, x_{i d}\right]=$ $\left[N_{P V}, N_{B A T}\right]$ randomly. $N_{P V}$ is the number of PV panel dan $N_{B A T}$ is number of battery. Initialize the solution of the problems generated using mathematical formulation as follows,

$$
x_{i}=\text { rand } * \text { (upper-range }- \text { lower-range) }+ \text { lower-range }
$$

where $x_{i}$ is the- $i^{\text {th }}$ individual candidate solution of the genetic algorithm, rand is a random number with uniform distribution in $[0,1]$, while the upper range and the lower range are the upper range and the lower range of PV panel and battery value.

3. Annual simulation was conducted to achieve the optimal configuration based on the proposed system.

4. Evaluation of the objective function: The evaluation processes in searching the total values of PV panels and battery were as follows:

a. The calculation of the fitness function value using Equation (5), based on the $N_{P V}$ and $N_{B A T}$ value obtained for each individual.

b. For each generation, the best fitness value can always be obtained. Generation process continues until meet the desired criteria stop and obtained the best fitness.

5. Selection process: Individuals selected in the next generation of the best individuals in the previous generation and compared for generations progress. The next generation is known to the child (offspring) is formed from the union of two individuals who acted as the current generation using crossover operators.

6. Crossing over: Crossover is the main genetic operator. Crossover allows the genes from different parents to be combined in children by exchanging materials between two parents. Cross over function randomly selects

Table 1. IT2FL rules.

\begin{tabular}{llll}
\hline Best Fitness & LOW & MEDIUM & HIGH \\
\hline$P_{\text {cross }}$ & HIGH & MEDIUM & LOW \\
$P_{\text {mut }}$ & LOW & MEDIUM & HIGH \\
\hline
\end{tabular}


a gene at the same coordinate from one of two parents and assign it to the child.

Mutation: Mutation was used to introduce some artificial diversification in population to avoid premature convergence to local optima. Uniform mutation method is used in this study.

\section{Simulation and Analysis}

Remote areas of the Rote Island were used for the case study in this study to design the PV-battery system simulation. Daily load demand characteristic in Rote Island is shown in Figure 6. This simulation used data per hour for one day and repeated for one year or 8760 hours. The time period of this project was 20 years. The value of inflation and interest rate established in this study were $8.17 \%$ and $8.25 \%$ based on the real conditions economics in Indonesia. The specification of PV panel, battery and inverter used in this study can be seen in Table 2 and 3. The parameters of GA used in this study are shown in Table 4. The crossover probability and the mutation probability were made adaptive using IT2FL.

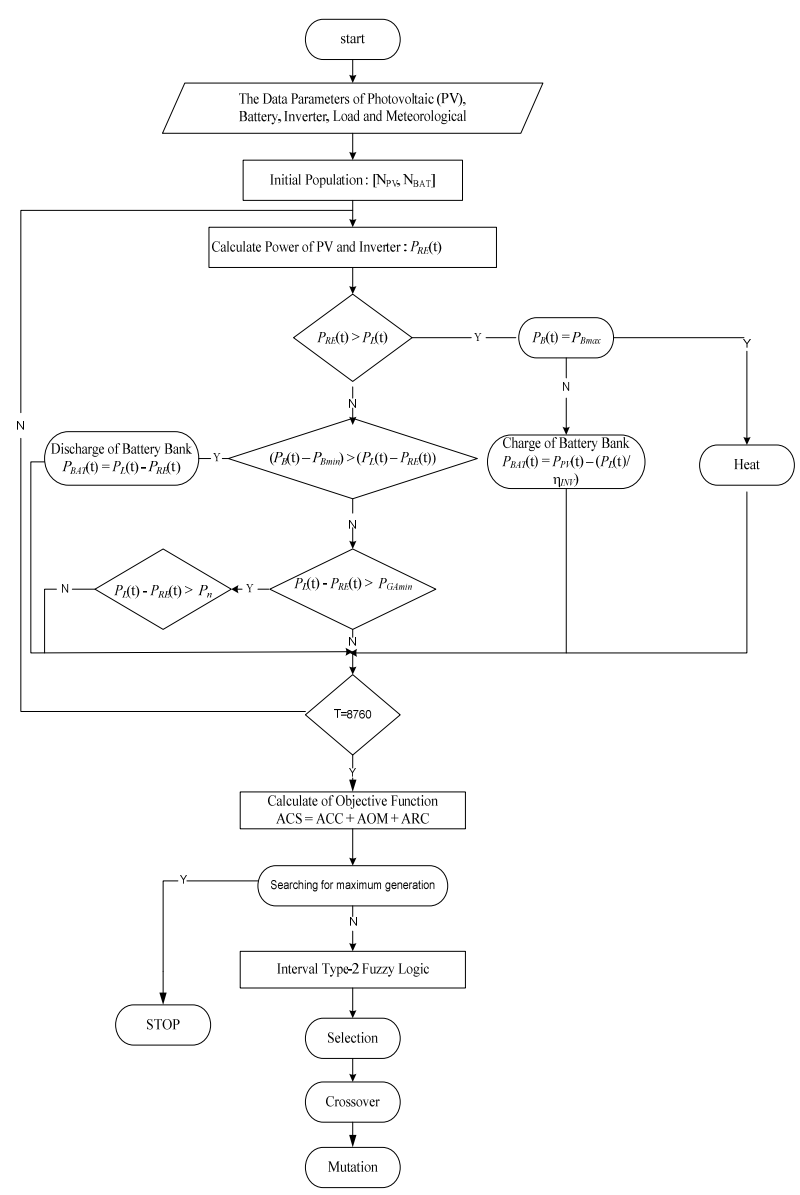

Figure 5. Flowchart of proposed method.

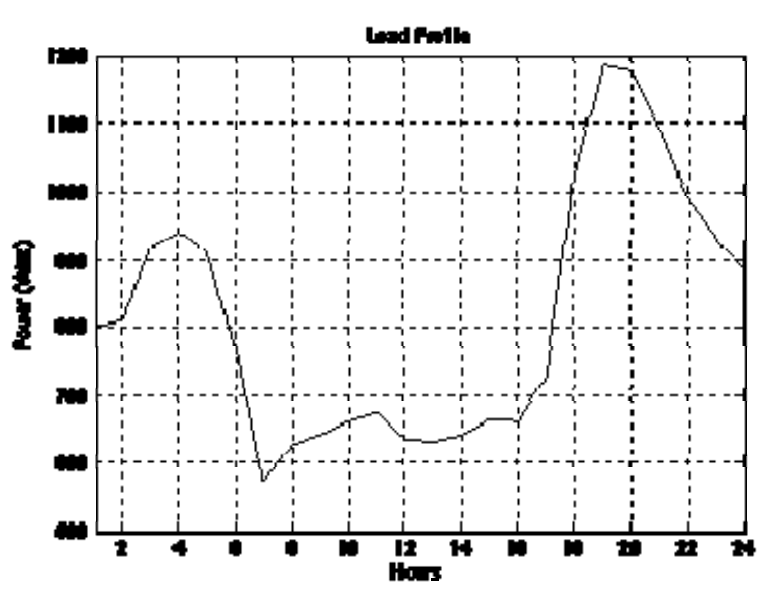

Figure 6. Load demand characteristic in Rote Island.

Table 2. Specification of PV panel [11].

\begin{tabular}{ll}
\hline Maximum output power & $120 \mathrm{~W}$ \\
\hline Efficiency & $90 \%$ \\
Area of single PV panel & $1.07 \mathrm{~m}^{2}$ \\
Capital cost & $\mathrm{US} \$ 230$ \\
Time periode of project & 20 years \\
\hline
\end{tabular}

Table 3. Specification of battery and inverter [11].

\begin{tabular}{ll}
\hline Battery & \\
\hline Maximum output power & $1.000 \mathrm{~W}$ \\
Capital cost & US\$ 400 \\
Replacement cost & US\$ 400 \\
Time periode of project & 10 years \\
Inverter & \\
Inverter capacity & $2000 \mathrm{~W}$ \\
Capital cost & US\$ 900 \\
Time periode of project & 20 years \\
\hline
\end{tabular}

Table 4. The parameter of GA.

\begin{tabular}{ll}
\hline Number of population & 30 \\
\hline Number of generation & 40 \\
\hline
\end{tabular}

The convergence speed of the proposed method was tested. Figure 7 shows the number of generation needed in order to converge to the best solution founded by respective algorithms required for the proposed method. The test results show that the proposed method convergence characteristic was better compared to others in terms of the required number of generations. Simulation result of PV-battery system which optimized using the proposed method and compared to two other methods can be seen in Table 5. 


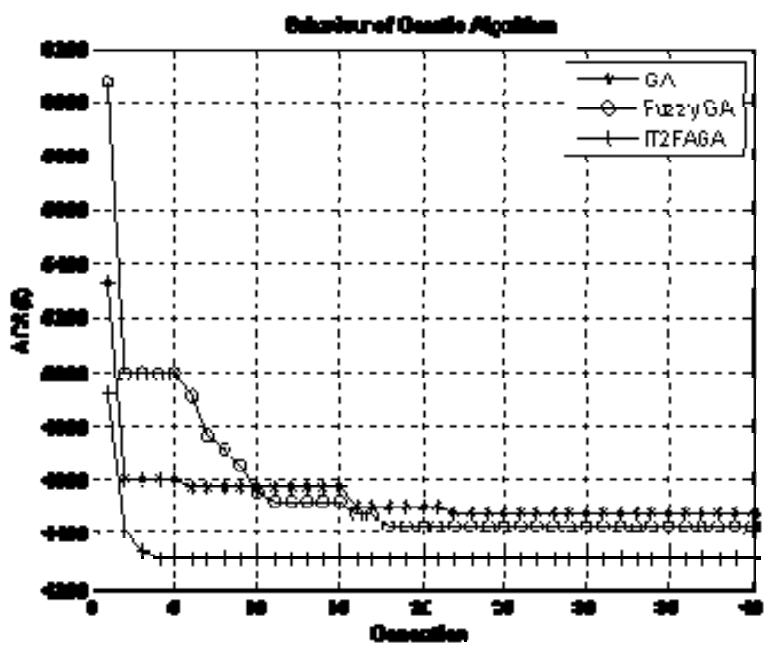

Figure 7. Convergence graphic of GA.

Tabel 5. Simulation result of PV-battery system design.

\begin{tabular}{lccc}
\hline Method & $\begin{array}{c}\text { Number of } \\
\text { Battery }\end{array}$ & $\begin{array}{c}\text { Number } \\
\text { of PV }\end{array}$ & $\begin{array}{c}\text { Annual Cost System } \\
\text { (US\$) }\end{array}$ \\
\hline SGA & 96 & 30 & 4483 \\
Fuzzy GA & 65 & 40 & 4429 \\
IT2FAGA & $\mathbf{8 9}$ & $\mathbf{3 0}$ & $\mathbf{4 3 1 3}$ \\
\hline
\end{tabular}

From Table 5 it can be seen that the optimization of PV-battery system using the proposed method can minimize the annual cost system (ACS) of US\$ 4,313 lower than two other methods

\section{Conclusion}

A new method for optimizing PV-Battery system based on interval type-2 fuzzy adaptive genetic algorithm (IT2FAGA) was proposed in this paper. The annual cost of system (ACS) is used as an objective function of the optimal PV-battery systems problem design. Test results indicate that the proposed method algorithm is efficiently found the optimal PV-battery system design, compared to Fuzzy GA and standard GA.

\section{Acknowledgements}

The authors acknowledge LPPM-ITS for giving financial support to this research via Laboratory Research Grant Scheme. The second author is very grateful to the Government of Indonesia, especially Directorate General of Higher Education (DIRJEN-DIKTI) for Excellency Scholarship awarded during his study in Graduate Program, Department of Electrical Engineering, Institut Technology Sepuluh Nopember (ITS), Surabaya, Indonesia. Last but not least, the authors are also very thankful to the Laboratory of Power System Simulation for all the - fa- cilities provided during this research.

\section{REFERENCES}

[1] H. M. Fargli, F. H. Fahmy, and M.A. H.EL-Sayed, "Artificial Intelligence Techniques for Controlling PVWind Powered Rural Zone in Egypt", International Conference on Renewable Energies and Power Quality (ICREPQ'09). Valencia (Spain), $15^{\text {th }}-17^{\text {th }}$ April, 2009.

[2] Goldberg DE. "Genetic Algorithms in Search, Optimization and Machine Learning", New York: Addison-Wesley; 1989.

[3] Orero SO, Irving MR, “A Genetic Algorithm Modeling Framework and Solution Technique for Short Term Optimal Hydrothermal Scheduling”, IEEE Trans Power Syst. 1998;13 (2):501-18

[4] Muhammad Abdillah, "Design of Interval Type 2 Fuzzy Logic Controller for DC-DC Zeta Converter", International Journal of Electrical Electronics Research \& Innovation (IJEERI), ISSN 2301-6132, Vol.2, No.1, March, 2013.

[5] Margo Pujiantara, Muhammad Abdillah, "Directional Over Current Relays (DOCRs) Coordination by Interval Type 2 Fuzzy Adaptive Particle Swarm Optimization (IT2FAPSO)", International Journal of Academic Research, Vol. 4. No. 3. May, 2012.

[6] Margo Pujiantara, Muhammad Abdillah, "Intelligent Over Current Relays Based on Interval Type 2 Fuzzy Logic Approach", Proceeding of 2012 International Conference in Green and Ubiquitous Technology, IEEE Catalog Number: CFP1256R-PRT, ISBN: 978-1-45772170-0, July $7^{\text {th }}-8^{\text {th }}$, Bandung, Indonesia, 2012.

[7] Adi Soeprijanto, Muhammad Abdillah, “Type 2 Fuzzy Adaptive Binary Particle Swarm Optimization for Optimal Placement and Sizing of Distributed Generation", Preceedings of The $2^{\text {nd }}$ International Conference on Instrumentation, Communication, Information Technology and Biomedical Engineering 2011, Indonesia, IEEE Catalog Number: CFP1187H-CDR, ISBN: 978-1-4577$1165-7$, Nopember $7^{\text {th }}-8^{\text {th }}, 2011$.

[8] H. Suryoatmojo, T. Hiyama, A. A. Elbaset, M. Ashari. "Optimal design of Wind-PV-Diesel-Batteray system using genetic algorithm", IEEJ. PE 2009; 129(3): 413-420.

[9] L.A.Zadeh, "The concept of a linguistic variable and its application to approximate reasoning-1", Information Science, vol.8, pp.199-249.

[10] A. B. M. Nasiruzzaman and M. G. Rabbani, "Implementation of Genetic Algorithm and Fuzzy Logic in Economic Dispatch Problem", The $5^{\text {th }}$ International Conference on Electrical and Computer Engineering ICECE 2008, 20-22 December 2008, Dhaka, Bangladesh.

[11] Firman Yudianto, " Optimal Design of Wind and PV Energy for Remote Areas Using Genetic Algorithm", Graduate Thesis, Electrical Engineering Department, Institut Teknologi Sepuluh Nopember, 2012. 